\title{
RIVER DISSOLVED OXYGEN PREDICTION BASED ON RANDOM FOREST AND LSTM
}

\author{
Juan Huan ${ }^{1, *}$, Bo Chen ${ }^{1}$, Xian Gen Xu' ${ }^{2}$, Hui Li ${ }^{1}$, Ming Bao Li ${ }^{1}$, Hao Zhang ${ }^{1}$
}

1 Changzhou University, Changzhou City, Jiangsu Province, China.

2 Changzhou Academy of Environmental Science, Jiangsu Province, China.

* Correspondence: huanjuan@cczu.edu.cn.

Highlights

- Random Forest (RF) and LSTM were developed for river DO prediction.

- $\mathrm{PH}$ is the most important feature affecting DO prediction.

- The model base on RF is better than the model not on RF, and the dimensionality of the input data is reduced by RF.

- RF-LSTM model is outperformed SVR, RF-SVR, BP, RF-BP, LSTM, RNN models in DO prediction.

\begin{abstract}
In order to improve the prediction accuracy of dissolved oxygen in rivers, a dissolved oxygen prediction model based on Random Forest (RF) and Long Short Term Memory networks (LSTM) is proposed. First, the Random Forest performs feature selection, which reduces the input dimension of the data and eliminates the influence of irrelevant variables on the prediction of dissolved oxygen. Then build the LSTM river dissolved oxygen prediction model to fit the relationship between water quality data and dissolved oxygen, and finally use real water quality data in the river for verification. The experimental results show that the mean square error (MSE), absolute error (MAE), mean absolute percentage error $(M A P E)$, root mean square error (RMSE), and coefficient of determination $\left(R^{2}\right)$ of the RF-LSTM model are 0.658, 0.528, 13.502, 0.811, 0.744, respectively, which are better than other models. The RF-LSTM model has good predictive performance and can provide a reference for river water quality management.
\end{abstract}

Keywords. Dissolved oxygen prediction, LSTM, Random forest, Time series, Water quality management.

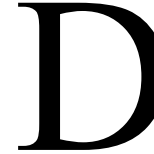

issolved oxygen (DO) concentration reflects the equilibrium between oxygen-producing (e.g., photosynthesis) and oxygen-consuming (e.g., aerobic respiration, nitrification, and chemical oxidation) processes in aquatic ecosystems (Olyaie et al., 2017). Dissolved oxygen imbalance can cause problems with fish and shrimp deaths and destroy the ecological environment of the river (Rahman et al., 2020). Therefore, the establishment of accurate and practical prediction models has very important economic value and practical significance.

In recent years, many scholars have done a lot of work in the prediction of dissolved oxygen content and made great

Submitted for review on 22 January 2021 as manuscript number ITSC 14496; approved for publication as a Research Article and as part of the Computer Modeling and Statistics for Agriculture Collection by the Information Technology, Sensors, \& Control Systems Community of ASABE on 8 June 2021 achievements (Liu et al., 2014; Yu et al., 2016; Kisi et al., 2020). Faruk (2010) combined the advantages of ARIMA for linear processing and neural network for nonlinear processing, proposed a hybrid model of ARIMA and neural network to predict water quality. Liu (2013) proposed a mixed dissolved oxygen content prediction model based on particle swarm optimization and the least square support vector model. Khudhur et al. (2020) designed an Artificial Neural Networks model to predict the DO and applied the model to predict the DO in the Shatt Al-Arab River. The study of Chen and Yu (2018) proposed a novel chaos-fireworks algorithm (FWA) based hybrid optimization algorithm and a neural network online soft-sensor model, which was to realize the rapid and accurate measurement of dissolved oxygen in wastewater.

The above methods prove that the fusion model is helpful to improve the prediction accuracy of dissolved oxygen, but they are all simple machine learning models. In the case of a large amount of data, they cannot fully fit the complex relationship between the data. In recent years, prediction methods based on recurrent neural networks have been 
widely used (Selvin et al., 2017; Li and Lin, 2018). Recurrent Neural Network (RNN) introduces the concept of timing into the network structure design, making it more adaptable in timing data analysis (Liu and Shen, 2019). As a variant model of RNN, LSTM effectively compensates for the memory attenuation problem of RNN (Quan et al., 2018). Nelson et al. (2017) studied the usage of LSTM networks and predicted future trends of stock prices based on the price history. Cortez et al. (2019) proposed the LSTM model to predict emergency events, and the result is presented to show the effectiveness of the LSTM architecture compared to traditional time series analysis and machine learning methods. According to the above paper analysis, the fusion model has better prediction performance than the single model, and the LSTM model has higher prediction accuracy than the traditional prediction model. Therefore, this research attempts to establish a more accurate LSTM fusion model in the prediction of dissolved oxygen in rivers.

Based on the above research, this article proposes a river dissolved oxygen prediction model based on random forest and LSTM. Random forest is used to calculate the feature importance scores of water quality factors, then feature selection, and finally use LSTM to perform dissolved oxygen prediction experiments. Experimental results show that the Random Forest algorithm can eliminate the influence of irrelevant variables on the prediction of dissolved oxygen. Compared with the LSTM, RNN, RF-BP, BP, RF-SVR, and SVR models, RFLSTM has the highest prediction accuracy and improves the river prediction accuracy of dissolved oxygen.

The rest of this article is organized as follows: Section 1 describes the study area, data collection, data preprocessing results. Section 2 introduces the Random Forest and LSTM algorithm, the prediction process of the RF-LSTM model. Section 3 uses the RF-LSTM model to predict the dissolved oxygen at $4,8,12$, and $16 \mathrm{~h}$ in the future and compares the performance of the RF-LSTM model with other prediction models. Section 4 describes the conclusions of this article.

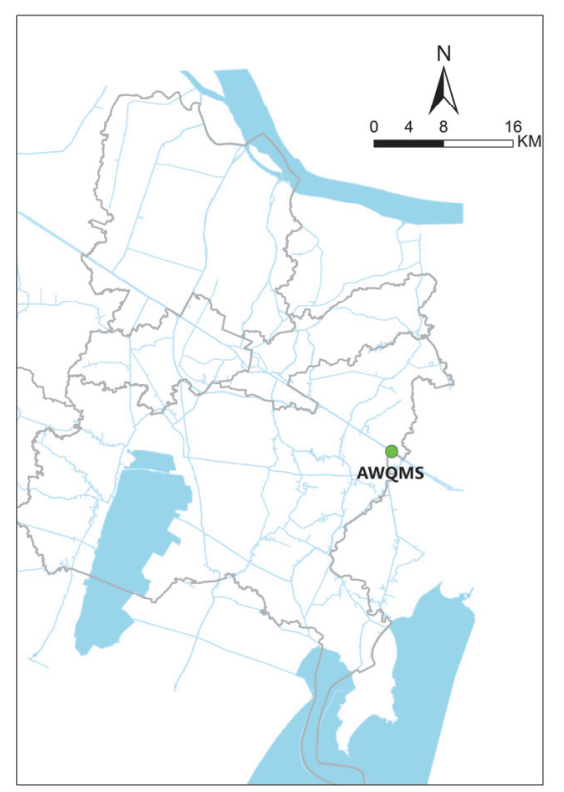

Figure 1. Location of automatic station.

\section{Study Area And Data Preprocessing}

\section{STUDY AREA}

The area studied is a river in the south of the Yangtze River in China, and the river area belongs to the northern subtropical monsoon climate with abundant rainfall. The river is equipped with an automatic water quality monitoring station (AWQMS), it is located at $120^{\circ} 7^{\prime} 21.87502 " \mathrm{E}$ and $31^{\circ} 41^{\prime} 11.78584^{\prime \prime N}$. Figure 1 shows the location of the automatic station.

\section{Data Preprocessing}

The data collection time in the experiment is from 1 January 2019 to 31 December 2019, and from 21 April 2020 to 6 July 2020 . The data is collected every $4 \mathrm{~h}$, there are 2636 records in total. Due to special reasons, the data from 1 January 2020 to 20 April 2020, cannot be obtained. The collected nine water quality features include Ammonia Nitrogen (AN), Total Phosphorus (TP), CODmn, Total Nitrogen (TN), PH, Water Temperature (WT), Dissolved Oxygen, Conductivity, and Turbidity. Figure 2 shows the trend change information of water quality features over time, and table 1 shows the statistics of water quality data.

From figure 2 and table 1, some data are abnormally high, abnormally low, or missing values. In addition, these indicators have different dimensions and magnitudes. To ensure the integrity and stability of the time series, it is necessary to preprocess the data.

\section{Missing Value Processing}

Our data contains 2636 samples, calculated in conjunction with table 1: The single water quality factor with the most missing values is 38 (Ammonia Nitrogen), accounting for about $1.5 \%$, so there are fewer missing data. Our data collection time is once every 4 hours, and the interval between missing data will not be very large. For the above reasons, we use linear interpolation to fill in missing data (Xu and Liu, 2012; Chen et al., 2018). The linear interpolation is:

$$
x_{d+i}=x_{d}+\frac{i \cdot\left(x_{d+j}-x_{d}\right)}{j}, 0<i<j
$$

Equation $1 x_{d+i}$ is the missing data at $d+i$ time, $x_{d}$ and $x_{d+j}$ is the original values at $d$ time and $d+j$ time, respectively. Part of the data processing before and after comparison is shown in figure 3.

\section{OUTLIER Processing}

The presence of very large or very small values in the data set indicates that there may be outliers, so these abnormal data must be processed. Box plot can be used to detect outliers, the calculation of the box plot is (Hubert and Vandervieren, 2008):

$$
\begin{gathered}
\mathrm{IQR}=Q 3-Q 1 \\
\text { UpperLimit }=Q 3+1.5 * I Q R \\
\text { LowerLimit }=Q 1-1.5 * I Q R
\end{gathered}
$$




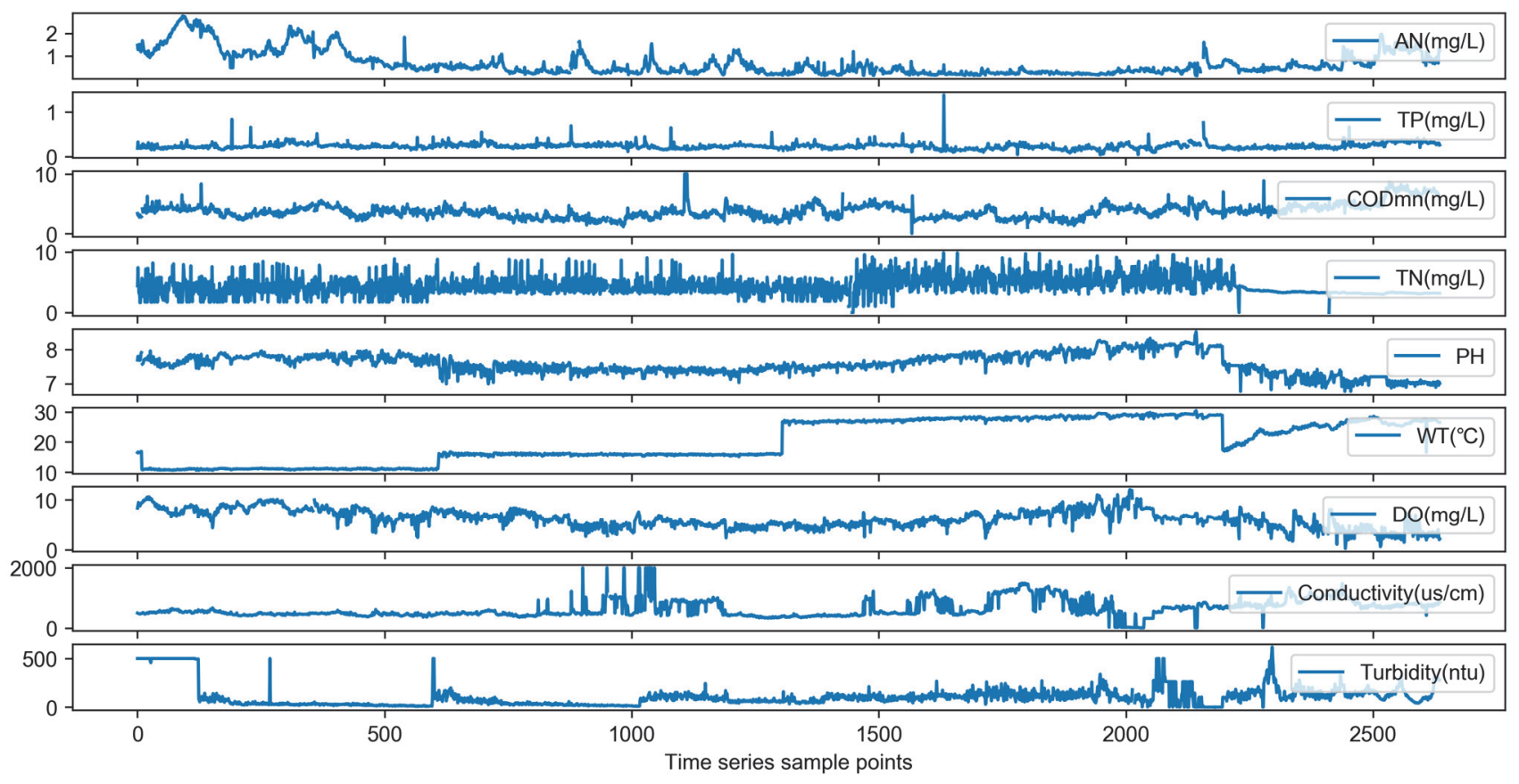

Figure 2. Time series data of water quality features.

Table 1. Water quality statistics information.

\begin{tabular}{cccccccccc}
\hline & $\begin{array}{c}\mathrm{AN} \\
(\mathrm{mg} / \mathrm{L})\end{array}$ & $\begin{array}{c}\mathrm{TP} \\
(\mathrm{mg} / \mathrm{L})\end{array}$ & $\begin{array}{c}\text { CODmn } \\
(\mathrm{mg} / \mathrm{L})\end{array}$ & $\begin{array}{c}\mathrm{TN} \\
(\mathrm{mg} / \mathrm{L})\end{array}$ & $\mathrm{PH}$ & $\begin{array}{c}\text { WT } \\
\left({ }^{\circ} \mathrm{C}\right)\end{array}$ & $\begin{array}{c}\text { DO } \\
(\mathrm{mg} / \mathrm{L})\end{array}$ & $\begin{array}{c}\text { Conductivity } \\
(\text { us/cm })\end{array}$ & $\begin{array}{c}\text { Turbidity } \\
(\mathrm{ntu})\end{array}$ \\
\hline count & 2599 & 2586 & 2608 & 2619 & 2615 & 2622 & 2619 & 2620 & 2623 \\
mean & 0.678 & 0.234 & 3.751 & 4.396 & 7.583 & 20.313 & 6.186 & 632.982 & 108.936 \\
std & 0.523 & 0.065 & 1.195 & 1.497 & 0.296 & 6.998 & 1.832 & 285.828 & 111.214 \\
min & 0.150 & 0.040 & 0.010 & 0.000 & 6.770 & 10.600 & 0.210 & 0.800 & 0.000 \\
0.25 & 0.310 & 0.197 & 2.900 & 3.340 & 7.390 & 15.600 & 4.885 & 445.100 & 35.400 \\
0.5 & 0.490 & 0.226 & 3.650 & 4.090 & 7.570 & 17.800 & 6.100 & 522.400 & 84.200 \\
0.75 & 0.850 & 0.262 & 4.340 & 5.230 & 7.800 & 27.400 & 7.510 & 780.550 & 128.950 \\
max & 2.750 & 1.389 & 10.000 & 9.850 & 8.520 & 30.400 & 12.060 & 1996.500 & 616.500 \\
\hline
\end{tabular}

Among them Q3 is the $75 \%$ quantile, Q1 is the $25 \%$ quantile, IQR is the upper and lower quartiles, UpperLimit is the upper boundary, and LowerLimit is the lower boundary. The specific box plot of water quality features is shown in figure 4 .

Among them, WT, Conductivity, and Turbidity are scaled between 0 and 15 . Values higher than the upper boundary and lower than the lower boundary are marked as outliers. The outliers will be filled as missing values and these missing values will be filled in by linear interpolation.

\section{NORMALIZATION PROCESSING}

In order to eliminate the influence of different magnitudes of water quality characteristics on the accuracy of model prediction, the water quality features are normalized between 0 and 1 , so that all water quality indicators are at the same magnitude. The normalization method (Munkhdalai et al., 2019) is:

$$
x^{\prime}=\frac{x-\min }{\max -\min }
$$

In equation $5, x^{\prime}$ is the normalized value, $x$ is the original value, $\min$ is the minimum value of the feature $x$, and $\max$ is

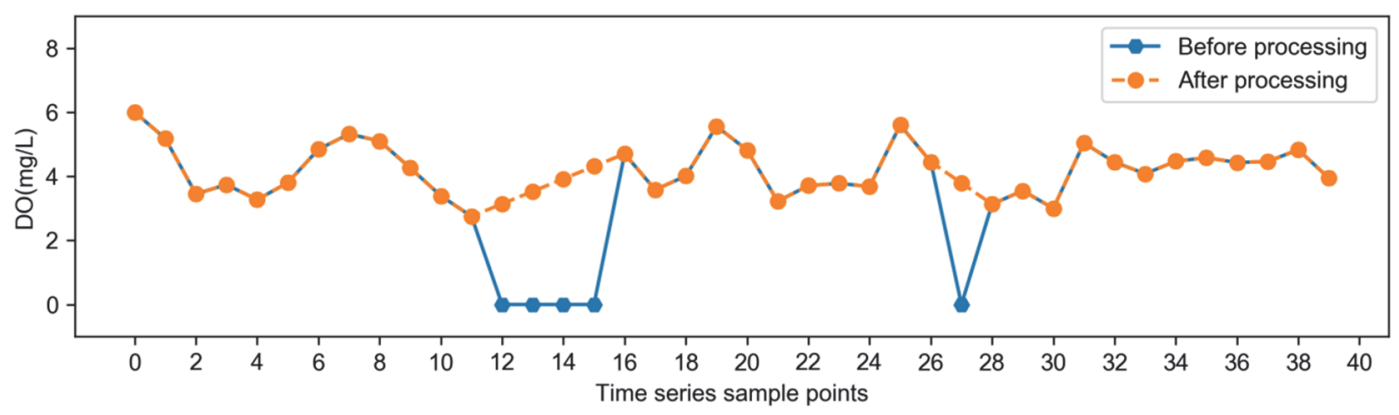

Figure 3. Missing value processing. 


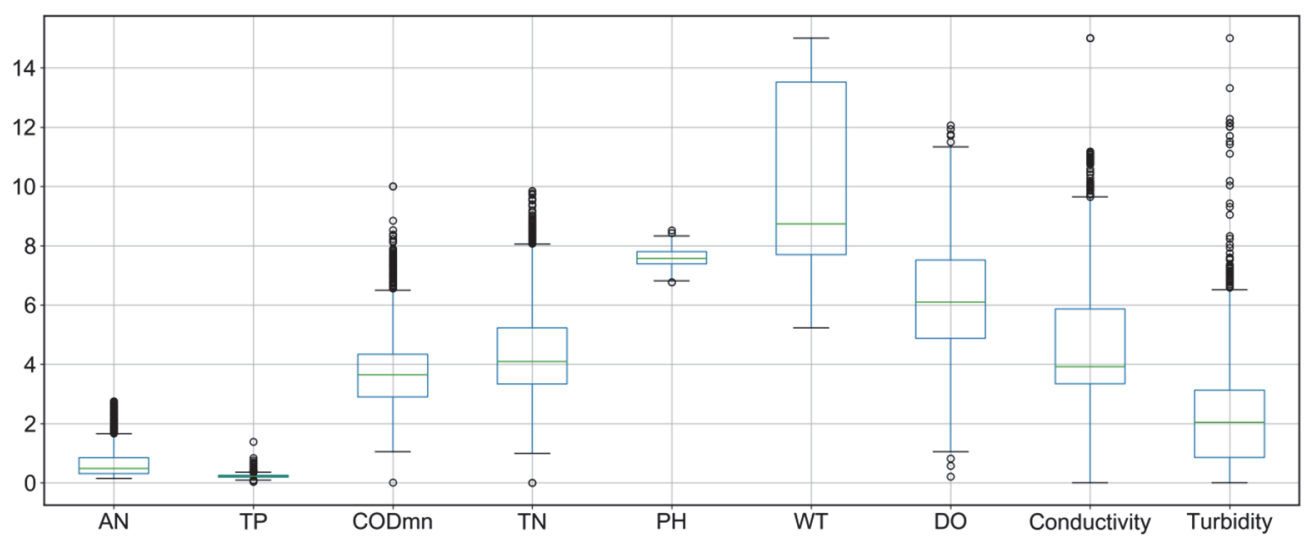

Figure 4. Box plot of water quality features.

the maximum value of the feature $x$. The data distribution after normalization is shown in figure 5, all water quality features are normalized between 0 and 1 .

\section{Prediction Model Construction RANDOM FOREST}

Random forest is a classifier based on multiple decision trees, and its output category result is determined by the mode of each tree output category (Ho, 1995). The Random Forest algorithm can be expressed as (Luo et al., 2019; Biau and Scornet, 2016):

\section{Random Forest algorithm:}

Input: Train Set $D_{n}$, number of tress $K>0$, number of features $M$. number of features selected $\mathrm{m} m<M$.

Output: Prediction of the random forest at $\mathrm{x}$.

for $b=1$ to $K$

select a bootstrap sample $\mathrm{n} *$ from $D_{n}$.

grow a random-forest tree $\mathrm{b}$ to the $\mathrm{n} *$, it is stipulated that the leaf node (ln) of b shall not be greater than $n_{\text {min }}$

while $\ln <n_{\min }$ do

Select $m$ features at random from the $M$ features

Calculate the best split method based on $m$ features

end

end
The purpose of feature selection is to select some of the features that have a greater impact on the final result from more features. Through feature selection, some feature variables that are irrelevant to the task or even redundant can be deleted. Simplifying the features will often result in a more accurate model (Luo et al., 2019). The Random Forest algorithm can measure the importance of features in regression or classification problems (Biau and Scornet, 2016), and perform feature selection based on the ranking. The specific steps of feature importance calculation are as follows (Tao and Du, 2019):

1. For each tree in the Random Forest, use out-of-bag data to calculate the out-of-bag error, denoted as errOOB1;

2. To measure the importance of the $j$-th feature, the $j$-th feature in the out-of-bag data is randomly scrambled, and its error is calculated again and denoted as errOOB2;

3. If there are $\mathrm{N}$ trees in the Random Forest, the importance score of feature $\mathrm{J}$ can be obtained from the average value on $\mathrm{N}$ trees by calculating the difference of out-of-bag error before and after the shuffling. The calculation equation for feature importance of feature $\mathrm{j}$ is:

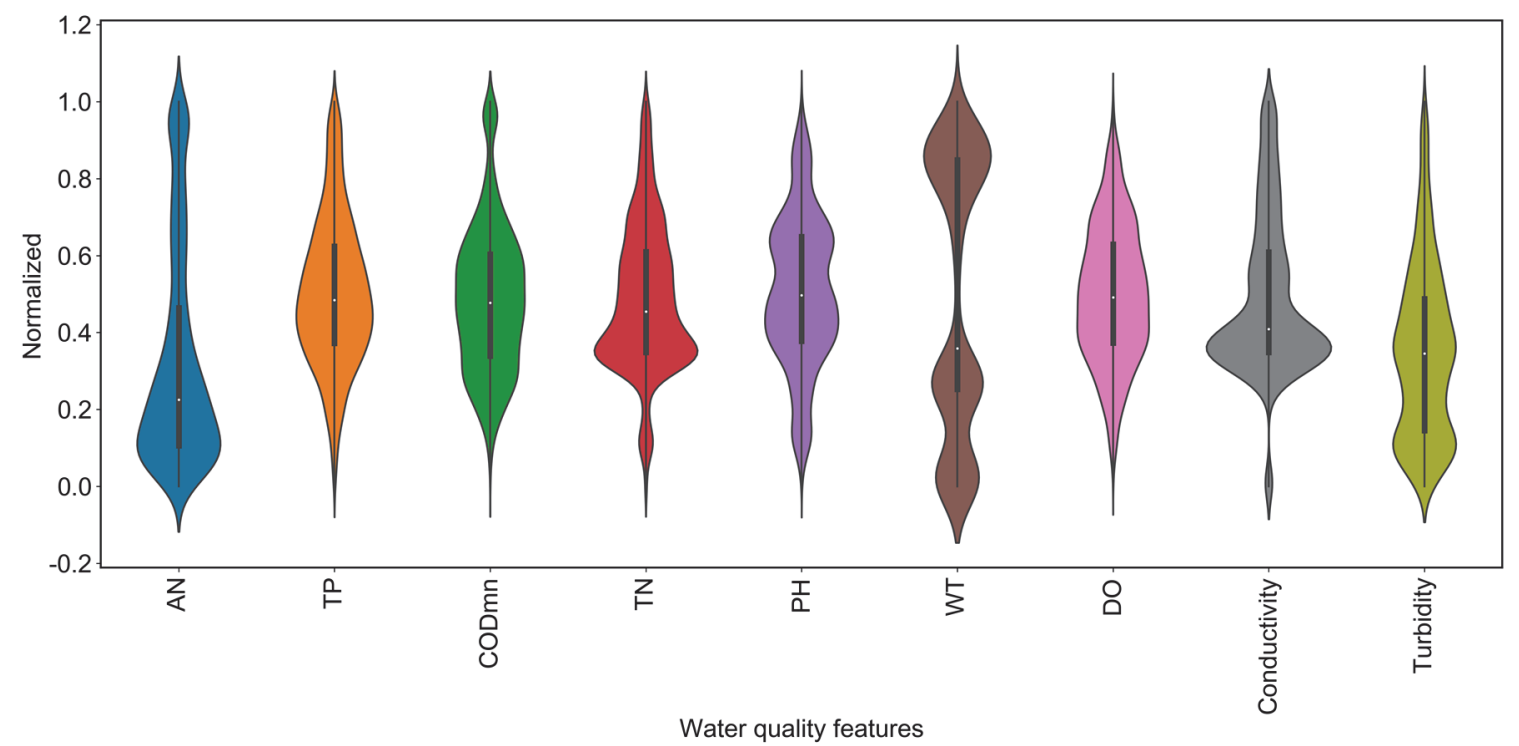

Figure 5. Normalized data distribution. 


$$
V I=\frac{\sum_{i=1}^{N}(e r r O O B 2-e r r O O B 1)_{i}}{N}
$$

\section{LSTM ALGORITHM}

LSTM Neural network is a kind of recurrent neural network (Zhu et al., 2020), which can overcome the problem of gradient disappearance in neural networks and can deeply dig the inherent laws in time series (Liu et al., 2020). On the basis of traditional RNN, the input and output of information and the state of cell units are controlled by adding forget gate, input gate, and output gate (Zhu et al., 2020). The basic network structure of LSTM is shown in figure 6.

The calculation process of the entire storage unit can be expressed by the following Steps:

Step 1, LSTM throws away some information from a sigmoid layer called the "forget gate layer."

$$
f_{t}=\sigma\left(W_{f} \bullet\left[h_{t-1}, x_{t}\right]+b_{f}\right)
$$

Step 2, LSTM decides what new information going to store in the cell state. First, the "input gate layer" decides which values will update by a sigmoid function. Second, a tanh layer creates a vector of new candidate values.

$$
\begin{gathered}
i_{t}=\sigma\left(W_{i} \bullet\left[h_{t-1}, x_{t}\right]+b_{i}\right) \\
C^{\prime}{ }_{t}=\tanh \left(W_{C} \bullet\left[h_{t-1}, x_{t}\right]+b_{C}\right)
\end{gathered}
$$

Step 3, LSTM uses $f_{t}, i_{t}, C^{\prime}$ to update the old cell state to the new cell state.

$$
\mathrm{C}_{\mathrm{t}}=f_{t} * C_{t-1}+i_{t} * C_{t}^{\prime}
$$

Step 4, LSTM decides what to output. First, the "output gate layer" decides some information to output by a sigmoid function. Second, LSTM put the cell state through tanh, and multiply it by the output of the "output gate layer."

$$
o_{\mathrm{t}}=\sigma\left(W_{o}\left[h_{t-1}, x_{t}\right]+b_{o}\right)
$$

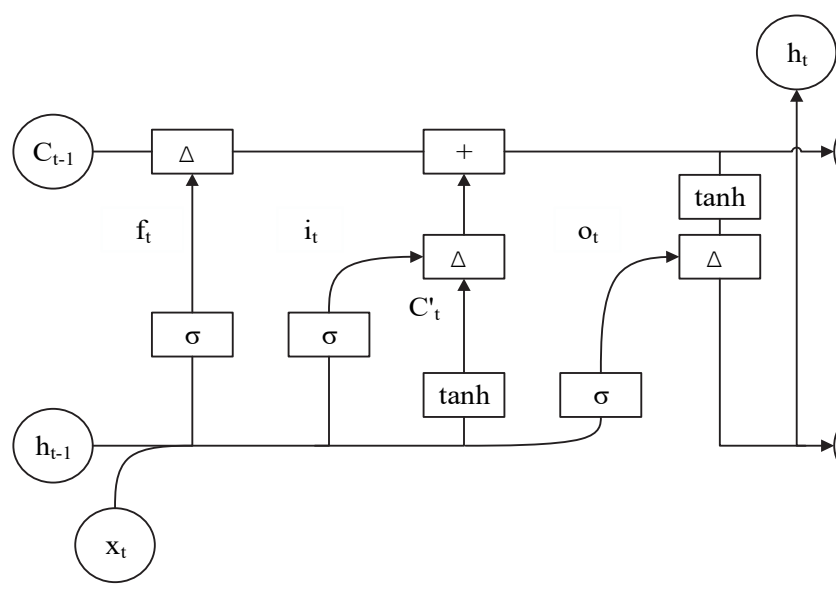

Figure 6. LSTM network structure.

$$
\mathrm{h}_{\mathrm{t}}=o_{t} * \tanh \left(C_{t}\right)
$$

where in $f_{t}, i_{t}, o_{t}$ are the $t$ time's output of forget gate, input gate, and output gate, $h_{t}$ is $t$ time's output of LSTM unit; $C_{t}^{\prime}$ is the candidate state of the input; $C_{t}$ is the memory unit state at $t$ time; $W, b$ a coefficient matrix and a bias vector; $\sigma$ is sigmoid activated function; $x_{t}$ is the $t$ time input (Altché and de La Fortelle, 2017; Selvin et al., 2017).

\section{Dissolved OXygen Prediction Model BaSEd ON RF- LSTM}

This article combines the two algorithms to build a dissolved oxygen prediction model based on Random Forest and LSTM neural network. The main process includes data preprocessing, feature selection by Random Forest, LSTM network training, prediction, and result in analysis. The specific flow is shown in figure 7.

1. Obtain water quality data through the automatic water quality station, and deal with abnormal and missing values on the collected data.

2. Normalize the data and use the Random Forest algorithm for feature importance calculation. Sort according to the importance of features and select the top-ranked features to reduce the influence of other features on the prediction of dissolved oxygen.

3. Divide the training set and the test set, and use the LSTM neural network for training until the constraint conditions are reached, and the prediction model of dissolved oxygen is obtained.

4. Use the test set to verify the dissolved oxygen prediction model, and compare and analyze with other models to obtain the optimal model for dissolved oxygen prediction.

\section{EXPERIMENTAL RESULTS AND ANALYSIS \\ RANDOM FOREST FEATURE SELECTION}

The selection of water quality factors that have a higher impact on the importance of dissolved oxygen will help improve the accuracy of dissolved oxygen prediction. This article takes Ammonia Nitrogen, Total Phosphorus, CODmn, $\mathrm{PH}$, Water Temperature, Conductivity, and Turbidity characteristics as input, and Dissolved oxygen as output. Select $10,50,100$ as the number of decision trees and 10, 50, 100 as the maximum tree depth to build a Random Forest regression model. The obtained water quality feature importance score is shown in table 2.

From the data in table 2, it can be seen that in the Random Forest model, the water quality factor feature importance scores are slightly different under a different number of decision trees and maximum tree depth, but the feature importance ranking is almost unchanged, which shows that Random Forest has certain stability in feature selection; the importance of $\mathrm{PH}$ feature is the highest, and the feature importance score is above 0.46 , which plays a decisive role in the prediction of dissolved oxygen; secondly, ammonia nitrogen and electrical conductivity are ranked higher, occupying the second and third places in the ranking; The ranking of water temperature and turbidity changes slightly under different parameters, but the overall scores are not far apart; 


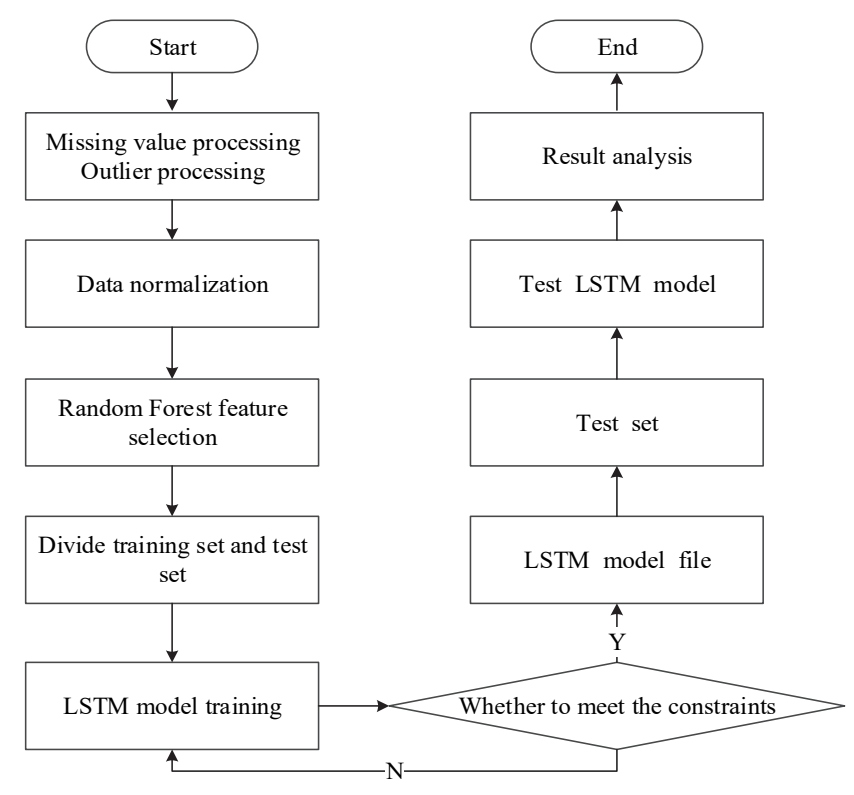

Figure 7. Dissolved oxygen prediction model process.

The importance scores of CODmn, Total Phosphorus, and Total Nitrogen are low and the ranking is the last three in order. Therefore, in the RF-LSTM model, discard these three water quality features.

\section{Dissolved OXYGen PREdiction BASEd ON RANDOM FOREST AND LSTM MODEL}

First, establish a dissolved oxygen prediction model according to the method proposed in Section 2.3. Then, select
PH, Conductivity, Ammonia Nitrogen, Turbidity, Water Temperature, and Dissolved Oxygen as the input parameters of the model, and take the Dissolved Oxygen at the time points of next $4,8,12$, and $16 \mathrm{~h}$ as the model output. Finally, setup LSTM parameters, the number of input layer nodes is 6 , the number of output layer nodes is 1 , the number of hidden layer nodes is 30 , the number of an epoch is 50 , the optimization algorithm is Adam, the learning rate is 0.001 , and the batch size is 32 .

Figure 8 shows the forecast results for 14 consecutive days. It can be seen from the figure that the prediction results at four different moments are not far from the true value, among which the $4 \mathrm{~h}$ prediction result is the closest to the true value, followed by 8,12 , and $16 \mathrm{~h}$. The time series sample points are in the interval from 5 to 30 , and the dissolved oxygen content changes little, and the prediction results are good. The time series sample points are between 30 and 45 , and the dissolved oxygen content rises rapidly after a sharp drop, and the prediction results deviate from the true value. The above shows that the four models have good prediction effects for dissolved oxygen sequences at stable moments, and poor prediction effects under abrupt changes, overall, the trend of the predicted results are consistent with the true value.

Figure 9 is the distribution of prediction error results at different time points. The figure contains 527 prediction error results. The four model prediction error results meet the normal distribution. Among them, the number of $4 \mathrm{~h}$ error results near 0 is the largest, followed by 8,12 , and $16 \mathrm{~h}$. With the increase of the absolute value of the error, the four prediction error distribution results are all decreasing, among

Table 2. Water quality feature importance score.

\begin{tabular}{|c|c|c|c|c|c|c|c|c|c|}
\hline $\begin{array}{l}\text { Maximum } \\
\text { Depth }\end{array}$ & $\begin{array}{l}\text { Number } \\
\text { of Trees }\end{array}$ & AN & TP & CODmn & $\mathrm{TN}$ & $\mathrm{PH}$ & WT & Conductivity & Turbidity \\
\hline 10 & 10 & 0.152 & 0.035 & 0.047 & 0.018 & 0.517 & 0.055 & 0.115 & 0.060 \\
\hline 10 & 50 & 0.151 & 0.035 & 0.050 & 0.015 & 0.508 & 0.066 & 0.116 & 0.059 \\
\hline 10 & 100 & 0.149 & 0.034 & 0.047 & 0.015 & 0.507 & 0.069 & 0.119 & 0.060 \\
\hline 50 & 10 & 0.142 & 0.042 & 0.054 & 0.023 & 0.462 & 0.079 & 0.128 & 0.072 \\
\hline 50 & 50 & 0.146 & 0.042 & 0.053 & 0.023 & 0.484 & 0.063 & 0.122 & 0.065 \\
\hline 50 & 100 & 0.147 & 0.043 & 0.056 & 0.024 & 0.473 & 0.069 & 0.123 & 0.065 \\
\hline 100 & 10 & 0.143 & 0.045 & 0.057 & 0.021 & 0.467 & 0.073 & 0.129 & 0.064 \\
\hline 100 & 50 & 0.147 & 0.044 & 0.056 & 0.022 & 0.472 & 0.069 & 0.123 & 0.067 \\
\hline 100 & 100 & 0.148 & 0.042 & 0.054 & 0.023 & 0.469 & 0.071 & 0.127 & 0.065 \\
\hline
\end{tabular}

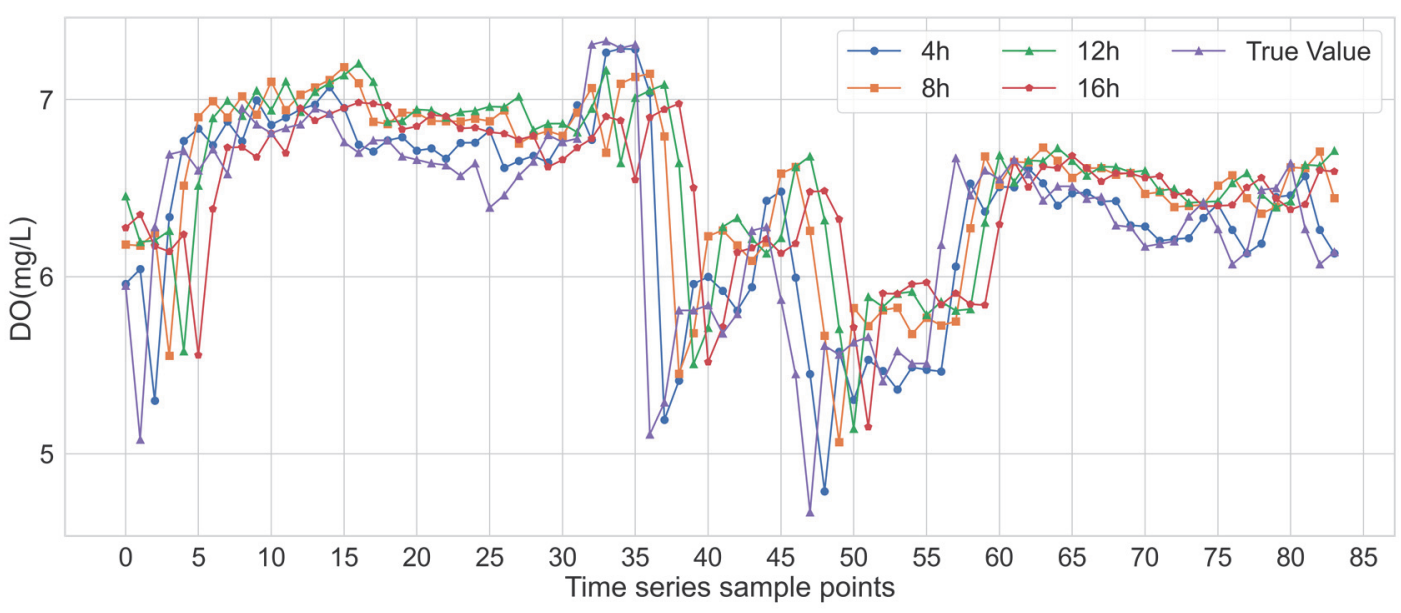

Figure 8. Prediction results of the RF-LSTM model at different time points. 
Table 3. RF-LSTM model prediction error table at different time points.

\begin{tabular}{cccccc}
\hline $\begin{array}{c}\text { Future Time } \\
\text { Points }\end{array}$ & MSE & MAE & MAPE & RMSE & R2 \\
\hline $4 \mathrm{~h}$ & 0.658 & 0.528 & 13.502 & 0.811 & 0.744 \\
$8 \mathrm{~h}$ & 1.083 & 0.719 & 19.397 & 1.040 & 0.579 \\
$12 \mathrm{~h}$ & 1.263 & 0.802 & 21.330 & 1.124 & 0.509 \\
$16 \mathrm{~h}$ & 1.280 & 0.780 & 20.164 & 1.131 & 0.502 \\
\hline
\end{tabular}

which the deceleration amplitude is the largest at $4 \mathrm{~h}$, followed by 8,12 , and $16 \mathrm{~h}$. The prediction error is basically between -1 and 1 , indicating that the model prediction results are good.

In order to show the prediction errors of the RF-LSTM model at different time points more intuitively, the error calculations are performed based on these 4 sets. Table 3 shows the model MSE, MAE, MAPE, RMSE, $\mathrm{R}^{2}$ obtained after the test. It can be seen from the table that the prediction error results in the next $4 \mathrm{~h}$ are the best, with the MSE, MAE, MAPE, RMSE, $\mathrm{R}^{2}$ being $0.658,0.528,13.502,0.811$, and 0.744 , respectively. As the forecast time increases, the forecast error is gradually increasing, but the error is within the acceptable range.

\section{Model COMPARISON ANALYSIS}

In order to verify the superiority of the proposed RFLSTM dissolved oxygen prediction model, here, we compare the LSTM model, RNN model, RF-BP model, BP model, RF-SVR model and SVR model. Adam optimizer usually has satisfactory performance (Ali et al., 2019; Arunsuriyasak et al., 2019), therefore, the comparison models in this article use Adam as the optimizer. In order to highlight the superiority of random forest, the same prediction model, both use the same parameters. Table 4 is the relevant parameters of each model.

Figure 10 shows the learning curves of the seven models under the parameters in table 4. From figures 10a, 10b, 10c, $10 \mathrm{~d}$, and $10 \mathrm{e}$, it can be seen that as the epoch increases, the loss value of the train set sample and the loss value of the validation set first to show a downward trend. It shows that when the Learning rate $=0.001$, the learning speed of the model is good. When epoch $>20$, the learning curve tends to be stable, and the fluctuation is small. It shows that 0.001 is
Table 4. Relevant parameters of each model.

\begin{tabular}{|c|c|c|c|c|c|c|}
\hline Model & $\begin{array}{l}\text { Hidden } \\
\text { Layers }\end{array}$ & $\begin{array}{c}\text { Learning } \\
\text { Rate }\end{array}$ & $\begin{array}{c}\text { Activation } \\
\text { Function }\end{array}$ & Epoch & Batch size & Optimizer \\
\hline$\overline{\text { RF-LSTM }}$ & 30 & 0.001 & $\tanh$ & 50 & 32 & adam \\
\hline LSTM & 30 & 0.001 & $\tanh$ & 50 & 32 & adam \\
\hline RNN & 30 & 0.001 & $\tanh$ & 50 & 32 & adam \\
\hline RF-BP & 30 & 0.001 & relu & 50 & 32 & adam \\
\hline BP & 30 & 0.001 & relu & 50 & 32 & adam \\
\hline RF-SVR & \multicolumn{6}{|c|}{ epsilon:0, loss:epsilon insensitive, $\max$ iter: 1000} \\
\hline SVR & \multicolumn{6}{|c|}{ epsilon:0, loss:epsilon_insensitive, $\max$ _iter: 1000} \\
\hline
\end{tabular}

used as the Learning rate and has stable learning performance. Secondly, epoch=50 is somewhat high, but it does not affect the prediction performance of the model. It can be seen from figures $10 \mathrm{f}$ and $10 \mathrm{~g}$ that with the increase of training samples, the training set loss value and validation set loss value of the RF-SVR and SVR models gradually become flat. The above shows that the seven models are all fitted for the relationship between water quality data and dissolved oxygen, the parameters provided in table 4 can be used as the basic parameters of the comparison model.

Figure 11 shows the prediction results of 7 models for 14 consecutive days. When the time series sample points are between 30 and 35, the prediction results of the BP model deviate significantly from the true value. When the time series sample points are between 1 to 30 and 60 to 84, RF -The predicted value of LSTM is closest to the true value. Most of the predicted values of LSTM, RNN, RF-BP, BP, RF-SVR, and SVR are higher than the real values, with BP being the most obvious, followed by RF-BP. Overall, the prediction results of RF-LSTM are the best.

From figure 12, it can be seen that the RF-LSTM model with the largest number of errors in the prediction error results near the value of 0 has about 58 sample points. The center point of the prediction error distribution of the RF-LSTM model and the LSTM model is closest to the value of 0 , indicating that these two prediction results of this model are the most stable. The error center of the prediction results of RFSVR, SVR, and RNN models is slight to the right, indicating that the prediction results are slightly worse than the LSTM model and the RF-LSTM model. The error center point of BP and RF-BP prediction results deviates from 0 , and the peak value is low, indicating the worst prediction result.

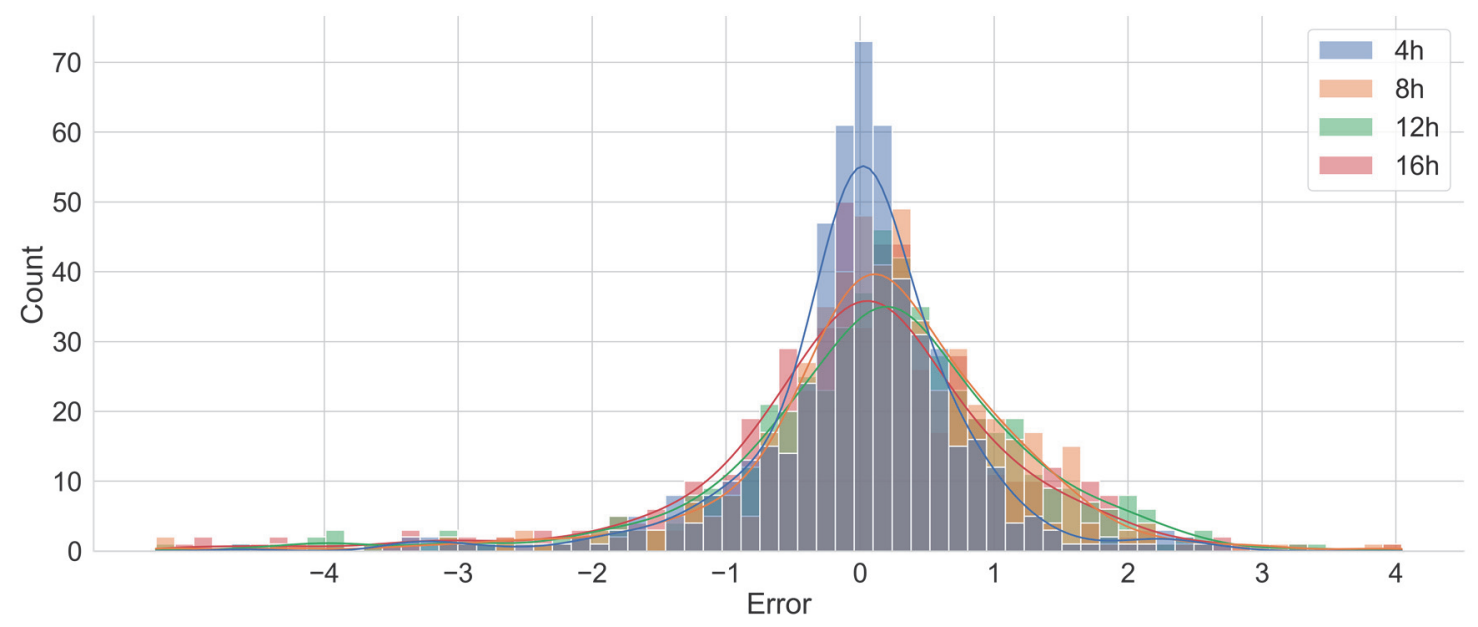

Figure 9. Distribution of prediction errors results at different time points. 


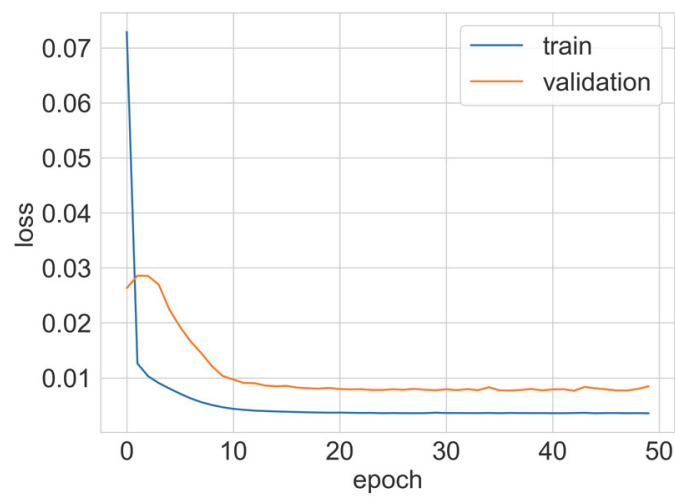

(a)

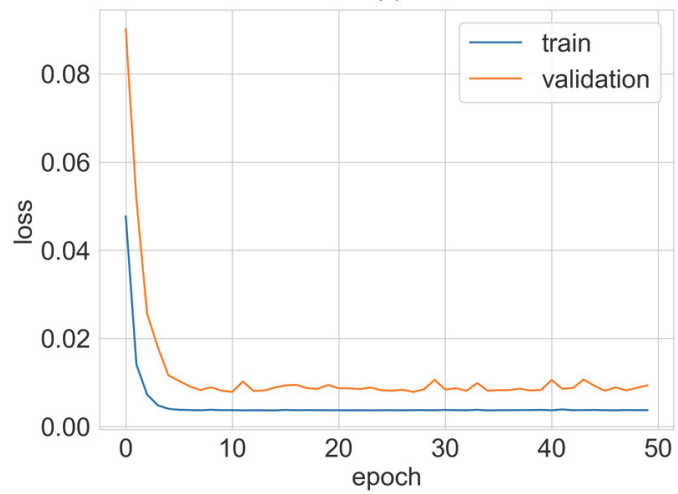

(c)

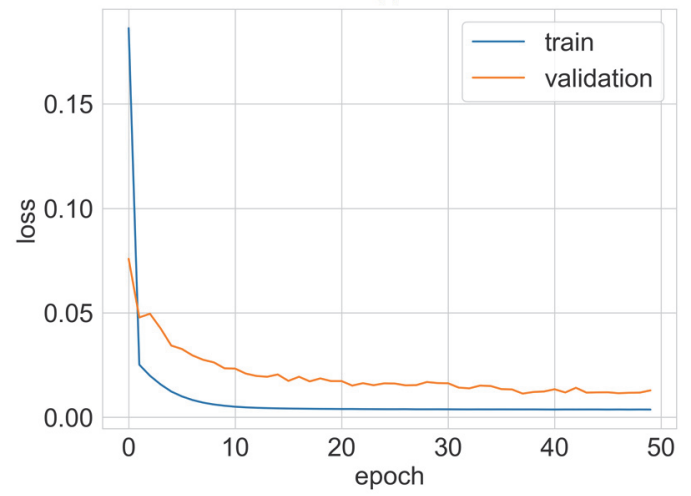

(e)

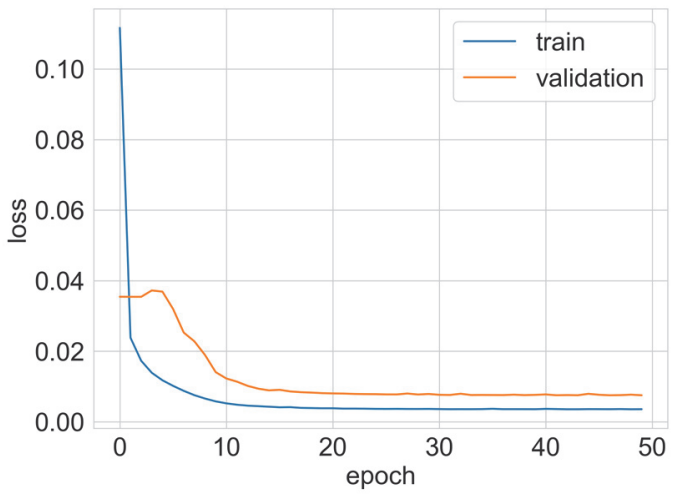

(b)

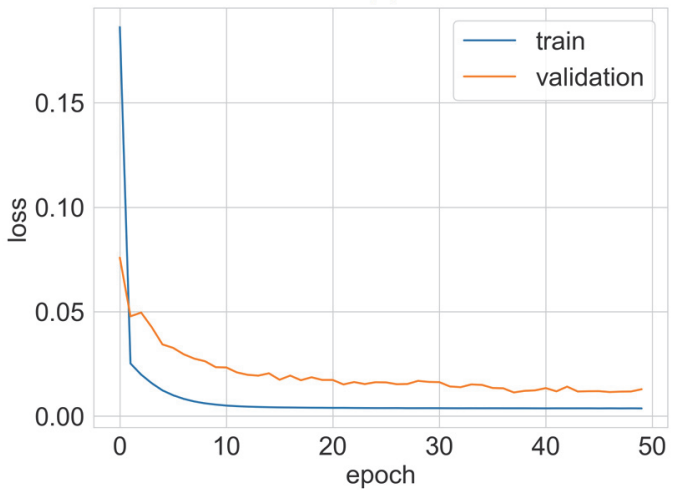

(d)

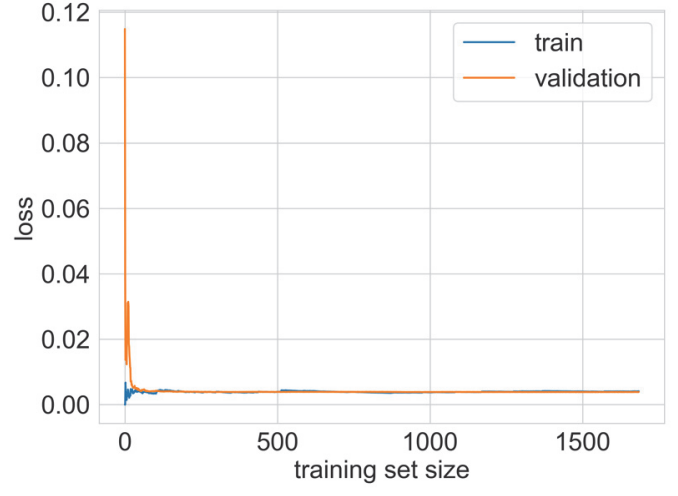

(f)

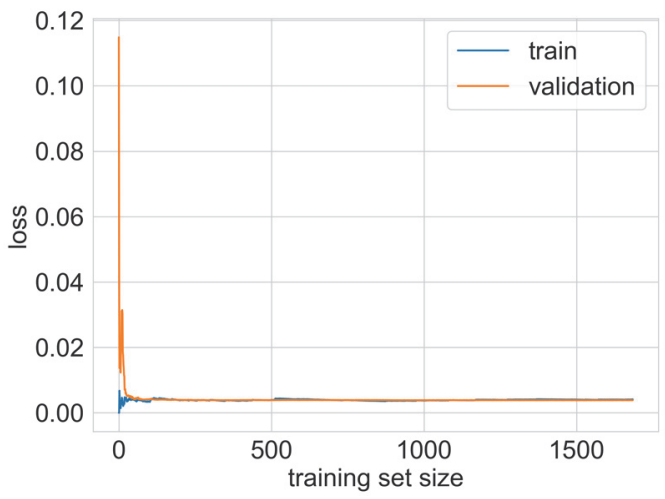

(g)

Figure 10. Seven kinds of model error loss information: (a) RF-LSTM, (b) LSTM, (c) RNN, (d) RF-BP, (e) BP, (f) RF-bp, (e) RF-SVR, (g) SVR.

It can be seen from table 5, the MSE, MAE, MAPE, RMSE, and $\mathrm{R}^{2}$ of LSTM are $0.670,0.534,13.756,0.818$, and 0.739 , respectively, which are more accurate than the prediction results of RNN, BP, and SVR. The above shows that
LSTM is more suitable for time series data forecasting. For the LSTM model, after random forest feature selection, the MSE, MAE, MAPE, RMSE, and $\mathrm{R}^{2}$ have improved perfor- 


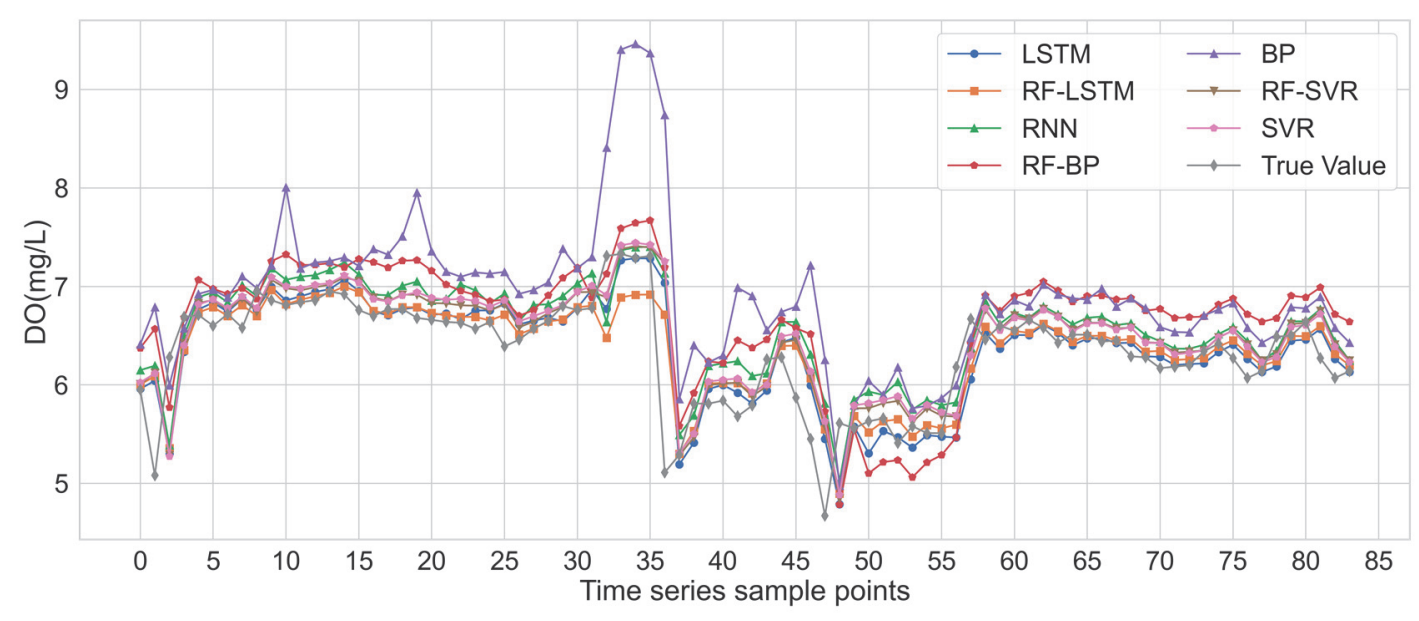

Figure 11. Trends of prediction results of different models.

mance by $1.79 \%, 1.12 \%, 1.84 \%, 0.85 \%$, and $0.67 \%$, respectively. For the BP model, after random forest feature selection, the MSE, MAE, MAPE, RMSE, and $\mathrm{R}^{2}$ have improved performance by $15.63 \%, 8.34 \%, 10.98 \%, 8.09 \%$, and $9.83 \%$, respectively. For the SVR model, after random forest feature selection, the MSE, MAE, MAPE, RMSE, and $\mathrm{R}^{2}$ have improved performance by $0.27 \%, 0.54 \%, 0.71 \%, 0.23 \%$, and $0.13 \%$, respectively. The above shows that the random forest's feature selection can eliminate the influence of irrelevant variables on the dissolved oxygen prediction and improve the prediction accuracy of the model. In addition, compared with other models, RF-LSTM has the lowest MSE, MAE, MAPE, and RMSE, and the highest $\mathrm{R}^{2}$, indicating that the RF-LSTM has the highest prediction accuracy.

\section{CONCLUSION}

Dissolved oxygen is a key influencing factor in rivers, so this article proposes a river dissolved oxygen prediction model based on random forest and LSTM. The main conclusions are:

1. Random Forest is used to calculate the key features affecting dissolved oxygen, and it is concluded that $\mathrm{PH}$ is the most important feature affecting dissolved oxygen. Feature factors with low feature importance are removed, and the dimensionality of the data is reduced.
Table 5. Predict errors of different models.

\begin{tabular}{cccccc}
\hline Model & MSE & MAE & MAPE & RMSE & R2 \\
\hline RF-LSTM & 0.658 & 0.528 & 13.502 & 0.811 & 0.744 \\
LSTM & 0.670 & 0.534 & 13.756 & 0.818 & 0.739 \\
RNN & 0.698 & 0.557 & 14.095 & 0.853 & 0.728 \\
RF-BP & 0.847 & 0.670 & 14.769 & 0.920 & 0.670 \\
BP & 1.004 & 0.731 & 16.592 & 1.001 & 0.610 \\
RF-SVR & 0.714 & 0.549 & 14.113 & 0.844 & 0.722 \\
SVR & 0.716 & 0.552 & 14.214 & 0.846 & 0.721 \\
\hline
\end{tabular}

2. The RF-LSTM model proposed in this article can effectively predict the dissolved oxygen value at the time points of the next $4 \mathrm{~h}$, with high prediction accuracy, but as the predicted time increases, the predicted accuracy is decreasing.

3. Compared with other models, the RF-LSTM model has a lower error and has improved the accuracy of dissolved oxygen prediction.

The river dissolved oxygen prediction model has good prediction performance and can provide guidance value for river water health, river ecology, and river water quality management.

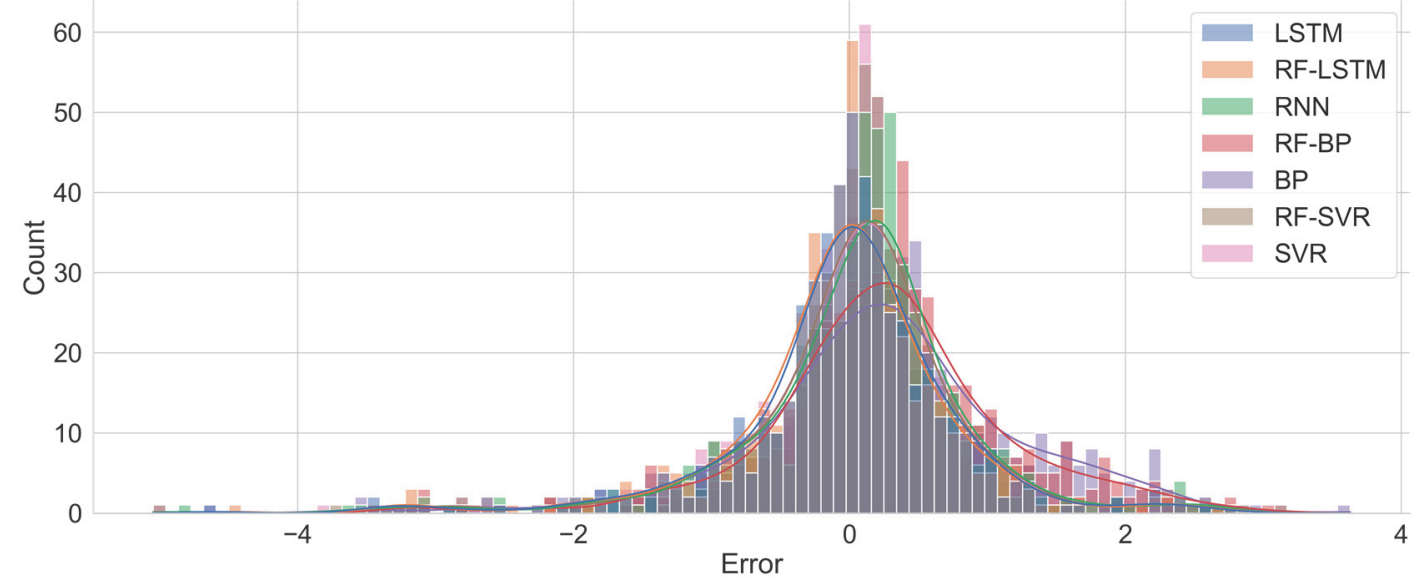

Figure 12. Distribution of prediction error results of different models. 


\section{ACKNOWLEDGEMENTS}

This study is funded by National Natural Science Foundation of China (61803050,52070023), and Changzhou Science and Technology Support Plan Project(CE20205037).

\section{REFERENCES}

Ali, M. N., Sarowar, M. G., Rahman, M. L., Chaki, J., Dey, N., \& Tavares, J. M. R. (2019). Adam deep learning with SOM for human sentiment classification. Int. J. Ambient Comput. Intelligence (IJACI), 10(3), 92-116. https://doi.org/10.4018/IJACI.2019070106

Altche, F., \& de La Fortelle, A. (2017). An LSTM network for highway trajectory prediction. Proc. 20th Int. Conf. on Intelligent Transportation Systems, (pp. 353-359). https://doi.org/10.1109/ITSC.2017.8317913

Arunsuriyasak, P., Boonme, P., \& Phasukkit, P. (2019). Investigation of deep learning optimizer for water pipe leaking detection. Proc. 16th Int. Conf. on Electrical Engineering/Electronics, Computer, Telecommunications and Information Technology, (pp. 85-88). https://doi.org/10.1109/ECTI-CON47248.2019.8955355

Biau, G., \& Scornet, E. (2016). A random forest guided tour. TEST, 25(2), 197-227. https://doi.org/10.1007/s11749-016-0481-7

Chen, R., \& Yu, J. (2018). Prediction of dissolved oxygen concentration in wastewater treatment process based on improved FWA-NN. China Environ. Sci., 38(10), 3739-3746.

Chen, Y. Y., Cheng, Q., Fang, X., Yu, H., \& Li, D. (2018). Principal component analysis and long short-term memory neural network for predicting dissolved oxygen in water for aquaculture. Trans. CSAE, 34(17), 183-191.

Cortez, B., Carrera, B., Kim, Y.-J., \& Jung, J.-Y. (2018). An architecture for emergency event prediction using LSTM recurrent neural networks. Expert Syst. Appl., 97, 315-324. https://doi.org/10.1016/j.eswa.2017.12.037

Faruk, D. O. (2010). A hybrid neural network and ARIMA model for water quality time series prediction. Eng. Appl. Artif. Intell., 23(4), 586-594. https://doi.org/10.1016/j.engappai.2009.09.015

Ho, T. K. (1995). Random decision forests. Proc. 3rd Int. Conf. on Document Analysis and Recognition. 1, pp. 278-282. IEEE.

Hubert, M., \& Vandervieren, E. (2008). An adjusted boxplot for skewed distributions. Comput. Stat. Data Analysis, 52(12), 5186-5201. https://doi.org/10.1016/j.csda.2007.11.008

Khudhur, Z. A., Dawood, A. S., \& Arab, S. A. (2020). Forecasting of dissolved oxygen in Shatt Al-Arab River based on parameters of water quality using artificial neural networks. Proc. 2nd Int. Conf. on Civil and Environmental Engineering Technologies. 888. IOP Publ. https://doi.org/10.1088/1757-899x/888/1/012049

Kisi, O., Alizamir, M., \& Docheshmeh Gorgij, A. (2020). Dissolved oxygen prediction using a new ensemble method. Environ. Sci. Pollut. Res., 27(9), 9589-9603. https://doi.org/10.1007/s11356019-07574-w

Li, J., \& Lin, Y. (2018). Time series data prediction based on multitime scale RNN. Comput. Appl. Softw., 35(7), 33-37.
Liu, J., \& Shen, G. (2019). Airline energy consumption sequence prediction based on LSTM. Comput. Appl. Softw., 36(10), 60-65.

Liu, S., Xu, L., Jiang, Y., Li, D., Chen, Y., \& Li, Z. (2014). A hybrid WA-CPSO-LSSVR model for dissolved oxygen content prediction in crab culture. Eng. Appl. Artif. Intell., 29, 114-124. https://doi.org/10.1016/j.engappai.2013.09.019

Liu, S., Xu, L., Li, D., Li, Q., Jiang, Y., Tai, H., \& Zeng, L. (2013). Prediction of dissolved oxygen content in river crab culture based on least squares support vector regression optimized by improved particle swarm optimization. Comput. Electron. Agric., 95, 82-91. https://doi.org/10.1016/j.compag.2013.03.009

Liu, X., Zhao, N., Guo, J., \& Guo, B. (2020). Prediction of monthly precipitation on the Tibetan Plateau based on LSTM neural network. J. Geo-Information Sci.(8), 1617-1629.

Luo, X., Hang, X., Cao, Y., Hang, R., \& Li, Y. (2019). Leading meteorological factors affecting cyanobacteria blooms in Taihu Lake under eutrophication conditions. Lake Sci., 31(5), 12481258.

Munkhdalai, L., Munkhdalai, T., Park, K. H., Lee, H. G., Li, D., \& Ryu, K. H. (2019). Mixture of activation functions with extended min-max normalization for forex market prediction. IEEE Access, 7, 183680-183691. https://doi.org/10.1109/ACCESS.2019.2959789

Nelson, D. M., Pereira, A. C., \& de Oliveira, R. A. (2017). Stock market's price movement prediction with LSTM neural networks. Proc. Int. Joint Conf. on Neural Networks (IJCNN), (pp. 1419-1426). https://doi.org/10.1109/IJCNN.2017.7966019

Olyaie, E., Zare Abyaneh, H., \& Danandeh Mehr, A. (2017). A comparative analysis among computational intelligence techniques for dissolved oxygen prediction in Delaware River. Geosci. Front., 8(3), 517-527. https://doi.org/10.1016/j.gsf.2016.04.007

Quan, B., Yang, B., Hu, K., Guo, C., \& Li, Q. (2018). Ship trajectory prediction model based on LSTM. Comput. Sci., 45(52), 126-131.

Rahman, A., Dabrowski, J., \& McCulloch, J. (2020). Dissolved oxygen prediction in prawn ponds from a group of one step predictors. Information Process. Agric., 7(2), 307-317. https://doi.org/10.1016/j.inpa.2019.08.002

Selvin, S., Vinayakumar, R., Gopalakrishnan, E. A., Menon, V. K., \& Soman, K. P. (2017). Stock price prediction using LSTM, $\mathrm{RNN}$ and CNN-sliding window model. Proc. Int. Conf. on Advances in Computing, Communications and Informatics, (pp. 1643-1647). https://doi.org/10.1109/ICACCI.2017.8126078

Tao, Y., \& Du, J. (2019). Long and short-term memory network temperature prediction based on random forest. Comput. Eng. Design, 40(3), 737-743.

Xu, L., \& Liu, S. (2012). Water quality prediction model based on APSO-WLSSVR. Shandong Univ. Eng. Sci, 42, 80-86.

Yu, H., Chen, Y., Hassan, S., \& Li, D. (2016). Dissolved oxygen content prediction in crab culture using a hybrid intelligent method. Sci. Rep., 6(1), 27292. https://doi.org/10.1038/srep27292

Zhu, Y., Gu, J., \& Meng, L. (2020). Photovoltaic power generation prediction model based on EMD-LSTM. Electric Power Eng. Technol., 39(2), 51-58. 